\title{
Uso de Software Colaborativo como Ferramenta de Apoio à Socialização do Conhecimento: Aplicação de um Estudo de Caso nos Correios usando o Telegram
}

\author{
Antonilson da Silva Alcantara ${ }^{1}$, Sandro Ronaldo Bezerra Oliveira ${ }^{1,2}$, Raimundo Viégas \\ Junior $^{2}$, Emanuel Amoras Rodrigues ${ }^{1}$ \\ 'Programa de Pós-Graduação em Ciência da Computação (PPGCC) - Universidade Federal \\ do Pará (UFPA) \\ ${ }^{2}$ Faculdade de Computação (FACOMP) - Universidade Federal do Pará (UFPA) \\ CEP 66075-110 - Belém - PA - Brasil \\ antonilsonalcantara@gmail.com, \{srbo,rviegas\}@ufpa.br, \\ emanorodrigues@gmail.com
}

\begin{abstract}
This paper presents the application of a Case Study in a House hold Distribution Center (CDD) at the Correios. This case study aims to analyze the use of the Telegram collaborative software as a tool to support the knowledge socialization in this sector of Correios. A brief presentation of the Case Study is made, followed by the description of the application in the organization. Finally, the results obtained by means a quantitative evaluation are presented, based on the data collected during the conduct of the case study, through the use of Telegram and also the company's management reports, followed by a qualitative evaluation based on the analysis of Strengths, Weaknesses, Opportunities and Threats from the perspective of the participants.
\end{abstract}

Resumo. Este trabalho apresenta a aplicação de um Estudo de Caso em um Centro de Distribuição Domiciliar (CDD) dos Correios. Esse estudo de caso visa analisar o uso do software colaborativo Telegram como ferramenta de apoio à socialização do conhecimento neste setor dos Correios. É feito uma breve apresentação do Estudo de Caso, seguida da descrição da aplicação na organização. Por fim, são apresentados os resultados obtidos por meio de uma avaliação quantitativa, com base nos dados coletados durante o a realização do estudo de caso, por meio do uso do Telegram e também dos relatórios gerenciais da empresa, seguida de uma avaliação qualitativa baseada na análise de Forças, Fraquezas, Oportunidades e Ameaças a partir da perspectiva dos participantes.

\section{Introdução}

A Gestão do Conhecimento é de grande importância dentro do cenário competitivo para as organizações. O conhecimento é apresentado como ativo empresarial, estabelecendo a capacidade das organizações competirem no mercado com um conjunto de capital intelectual bem estruturado, fruto de um bom processo de gestão desses ativos de conhecimentos, onde são gerados e armazenados (Aires et al., 2017).

Segundo Sehn et al. (2013), o conhecimento não é algo novo, porém é novo o conhecimento desde enquanto ativos corporativos e a percepção da necessidade de coordenálos ou geri-los com os cuidados iguais que os demais ativos tangíveis da organização.

Embora muitas organizações defendam o valor de captar conhecimento, elas geralmente estão mais interessadas no atual projeto de trabalho, do que documentar o que já foi realizado (Schafermeyer e Hoffman, 2016). Assim, mesmo o conhecimento tendo ganhado tamanha importância, muitas organizações negligenciam ou desconhecem uma forma de gerir esse ativo intelectual (Aires et al., 2017). 
Ferramentas colaborativas surgem como aliadas desse cenário, pois têm sido incorporadas pelas organizações com o objetivo de manter acessível o conhecimento. $\mathrm{O}$ uso de uma ferramenta colaborativa possibilita a integração, promove o gerenciamento e o compartilhamento de toda informação gerada e armazenada pela organização (Silva et al., 2016). Desta forma, este trabalho tem como objetivo responder a seguinte Questão de Pesquisa (QP): $O$ uso do Software Colaborativo Telegram auxilia no processo de socialização do conhecimento? Para isso, tem-se como hipótese básica: Os softwares colaborativos influenciam diretamente na eficácia da Gestão do Conhecimento. Como forma de responder essa questão, apresentamos os resultados de uma pesquisa a partir da aplicação em um estudo de caso participativo do software colaborativo Telegram, como ferramenta motivadora para o processo de socialização do conhecimento. Importante citar que na seção 3.2 deste trabalho tem-se a justificativa da escolha desta ferramenta, bem como na seção 3.3 pode ser encontrado um melhor detalhamento da metodologia usada para execução do estudo de caso.

Além desta seção introdutória, o artigo está organizado da seguinte maneira: a Seção 2 apresenta a fundamentação teórica; a Seção 3 apresenta o desenvolvimento do estudo de caso; a Seção 4 apresenta a Análise Quantitativa e a Análise Qualitativa dos resultados obtidos com o estudo de caso conduzido; e a Seção 5 apresenta as considerações finais, contribuições, limitações e trabalhos futuros deste trabalho.

\section{Fundamentação Teórica}

Segundo Braquehais (2017), conhecimento é a informação combinada com experiência, contexto, interpretação e reflexão, que pode ser usado para a tomada de decisão e para a ação. $\mathrm{O}$ conhecimento assume duas formas de demonstração: o conhecimento explícito, que rápido transfere-se às pessoas e se revela formal e diligentemente rápido, em palavras, números, dados, fórmulas, sons etc.; e o conhecimento tácito, que se mostra como o conhecimento fortemente firmado nas ações e vivências particulares das pessoas. Por essa característica, o conhecimento tácito é tido como altamente pessoal e dificultoso em protocolizar (Sehn et al., 2013).

Segundo Tabares et al. (2016), a gestão do conhecimento é a forma como os recursos humanos ou o aprendizado de máquina compartilham e adquirem experiências de diferentes fontes. A organização pode focar no conhecimento explícito dando ênfase na captura, no armazenamento e no compartilhamento deste conhecimento, ou focar no conhecimento tácito dando ênfase no compartilhamento do conhecimento a partir de interações entre os membros. Contudo, é necessário levar em consideração tanto o fator tecnológico quanto o humano para poder escolher qual desses estilos melhor adequa-se aos seus processos. Ferramentas colaborativas surgem como aliadas desse cenário, uma vez que têm sido incorporadas pelas organizações com o objetivo de manter acessível o conhecimento (Silva et al., 2016).

O aprendizado colaborativo, potencialmente, melhora o aprendizado e o desempenho dos participantes e aumenta sua motivação para realizar tarefas em grupo, que contribui, por sua vez, para o aumento da autoestima e para o desenvolvimento de habilidades do trabalho em equipe (Almenara et al., 2016).

\subsection{Trabalhos Relacionados}

Correa et al. (2017) buscaram identificar quais tipologias de ferramentas de apoio à gestão do conhecimento são utilizadas por uma empresa de tecnologia da informação, e como esta organização mensura o resultado obtido por esta utilização. Dentre as ferramentas identificadas existem softwares colaborativos, porém o trabalho trata de uma pesquisa descritiva, com abordagem qualitativa, de estudo de caso com entrevista aberta, porém não relata os benefícios do uso de software colaborativo no contexto da gestão do conhecimento. 
Souza e Heis (2017) relatam uma experiência de uso de uma ferramenta de discussões, aprendizados e avaliações de caráter colaborativo, com o objetivo de potencializar e oportunizar maior capacitação e preparo a uma equipe, construindo novos conhecimentos e compartilhando os já existentes. São analisadas algumas características da empresa e serviços de mercado fornecidos por ela para aplicar a ferramenta de aprendizagem colaborativa. Entretanto, o trabalho não apresenta os resultados e as discussões obtidos com esta experiência.

\section{Desenvolvimento do Estudo de Caso}

Esta seção relata em detalhes o contexto e o cenário onde o estudo de caso descrito neste trabalho foi conduzido.

\subsection{Sobre os Correios}

Como a maior empresa pública do Brasil, a Empresa Brasileira de Correios e Telégrafos (ECT), neste trabalho tratada como Correios, conta com mais de 105 mil empregados e estão presentes nos 5.570 municípios. Os Correios são, atualmente, uma empresa pública de direito privado, vinculada ao Ministério da Ciência, Tecnologia, Inovações e Comunicações (MCTIC). Tiveram sua origem no Brasil em 25 de janeiro de 1663 (Correios, 2019).

A Portaria $\mathrm{n}^{\circ}$ 6.194, de 27 de dezembro de 2016 do MCTIC (MCTIC, 2016) estabelece a meta de $93 \%$ de entrega, a serem alcançados em todas as modalidades de entrega, sendo respeitado, ainda, os prazos correspondentes a cada modal. Já a Portaria $\mathrm{n}^{\mathrm{o}} 1.203$, de 7 de março de 2018 do MCTIC institui alguns dos serviços de entrega dos Correios como prioritários, sendo base para os indicadores de desempenho das AC (Agências de Correios, com entrega externa) e dos CDD (Centros de Distribuição Domiciliar). São eles: Serviço de Entrega Expressa (SEDEX); Telegrama; Express Mail Service (EMS); Malote; Remessa Expressa; Logística Reversa; Objetos para entrega interna, como Caixa Postal e Posta Restante; Objetos destinados a grandes usuários; Serviços que venham a ser criados com características semelhantes ao de objetos qualificados (MCTIC, 2018).

\subsection{Sobre o CDD Mangueirão}

O CDD Mangueirão é uma unidade de distribuição que abrange nove bairros da cidade de Belém do Pará (Tapanã, Coqueiro, Parque Verde, Cabanagem, Una, Mangueirão, Bengui, São Clemente e Pratinha). Possui 40 distritos postais, na modalidade DDA (Distribuição Domiciliar Alternada), onde existem 80 áreas de entrega e 2 distritos na modalidade especial.

Na modalidade DDA, segundo o MCTIC (2018), divide-se a entrega externa em duas etapas, de forma que a distribuição da correspondência ocorra nestas etapas em dias úteis alternados, ou seja, cada distrito (área de entrega para um carteiro) possui duas áreas de entrega $\mathrm{A}$ e $\mathrm{B}$, sendo que no dia que entregar na área $\mathrm{A}$, a área $\mathrm{B}$ fica parada, e vice versa. $\mathrm{Na}$ área que ficar parada, todos os objetos classificados como prioritários, obrigatoriamente, devem ser entregues pelo Distrito Especial.

As entregas são realizadas com o apoio de Smartphone da empresa que possui rede móvel, porém algumas funções desse aparelho são desativadas. Não é possível realizar ligações dos mesmos por não possuir um número vinculado ao aparelho.

\subsubsection{Características dos Distritos}

Segundo Pimentel et al., (2012), os bairros da zona norte, que compõem a cidade de Belém, têm uma característica em especial pois apresentam uma função eminentemente residencial, habitada por uma classe pobre e que se caracteriza por uma ocupação de estrutura considerada desordenada, denotada por suas ruas tortuosas, com matos e água empossada.

Desta forma, os logradouros que compõem os distritos do CDD Mangueirão então contidos nessa região de Belém e possuem essas características, citadas anteriormente, requerendo os conhecimentos dos chamados "especialistas" (carteiro titular do distrito que 
conhece a maioria das pessoas e a numeração das residências na sequência irregular). Para tanto é necessária uma forma de manter todos esses especialistas acessíveis para os demais, mantendo uma rede de especialistas que possam ser consultados a qualquer momento e em qualquer lugar. Realizar ligações para o celular pessoal é inviável, pois os funcionários são orientados a não levar pertences pessoais durante a entrega. Assim, propôs-se o uso da ferramenta colaborativa Telegram, como meio de integração e comunicação entre os diferentes especialistas, por um período de um mês.

O Telegram foi lançado em 14 de agosto de 2013 e a versão Android foi lançada oficialmente em 20 de outubro de 2013. Ele usa uma plataforma aberta e é: "um aplicativo de mensagens com foco em velocidade e segurança, é super rápido, simples e gratuito" (Telegram.org, 2019). As características do Telegram foram fundamentais para sua escolha como software colaborativo para esse estudo de caso, destacando-se, principalmente, a simplicidade de uso, que induz o aprendizado do uso da ferramenta de forma intuitiva, dispensando, assim, a necessidade de curso de capacitação para manuseio do mesmo.

\subsection{Descrição do Estudo de Caso}

Esse estudo de caso deu-se em um CDD da região metropolitana de Belém, cujas características e particularidades oportunizaram a análise do impacto do uso de um software colaborativo como ferramenta de comunicação e socialização do conhecimento. Esse estudo de caso ocorreu entre os dias 30 de setembro e 31 de outubro de 2019, com um total de 5 participantes, sendo: 1 gerente de CDD, que atuou como apoio na base, para o caso de emergência; e 4 carteiros de Distritos Especiais (que atuam na entrega de objetos de prazos urgentes). O CDD Mangueirão foi selecionado por conveniência, onde havia uma facilidade de aproximação com as lideranças desse setor da organização e receptividade por parte do grupo para a execução do estudo.

Ao final do estudo de caso, houve uma reunião de feedback participativo, focus group, com os integrantes, no intuito de coletar informações, críticas e melhorias sobre o estudo de caso. Essa reunião de feedback foi conduzida por um aluno de doutorado, que se utilizou da matriz SWOT para fazer a análise qualitativa. A ferramenta SWOT (Strengths, Weaknesses, Opportunities e Threats), é usada para fazer análise de cenário ou análise de ambiente utilizado para definir o posicionamento estratégico de uma empresa (Santos et al., 2010).

$\mathrm{O}$ estudo de caso foi baseado em uma questão de pesquisa já mencionada anteriormente: QP - O uso do Software Colaborativo Telegram auxilia no processo de socialização do conhecimento? Para a realização desse estudo de caso, foram seguidos os seguintes passos: (i) definição da questão de pesquisa; (ii) escolha do software colaborativo; (iii) instalação do Telegram; (iv) definição e apresentação das regras da usabilidade do Telegram; (v) avaliação do uso do Telegram por meio de uma análise quantitativa; (vi) análise de feedback com os participantes do estudo de caso, para a obtenção dos dados qualitativos.

\section{Resultados e Análises}

Esta seção apresenta uma análise dos resultados quantitativos e qualitativos no estudo de caso.

\subsection{Resultados Quantitativos}

O relatório gerencial é um produto de um sistema de gestão usado na empresa, foco do estudo de caso, que retrata o desempenho de uma determinada unidade em relação aos indicadores estipulados pela organização.

O relatório pode ser consultado de duas formas: relatório detalhado, onde apresenta os dados referentes a cada indicador específico, vide Figura 1, representado visualmente por cores (sendo: azul, acima da meta; verde, dentro da meta; amarelo, abaixo da meta; e vermelho, muito abaixo da meta); e relatório geral, que apresenta a média final, calculado com base nos indicadores específicos, gerando uma nota final, vide Figura 2. 
No relatório detalhado são analisados quatro tipos de serviços prestados pelos correios, que são: Remessa Expressa; Remessa Econômica; Carta Registrada; e, Telegrama. Todos possuem prazo de urgência. Em cada um desses serviços são analisados três indicadores, que são: Percentual de entrega dentro do prazo (colunas REX, REC, CRG, TELG), que representa a quantidade de objetos entregues dentro do prazo estabelecido pela postagem do objeto pelo cliente, sendo importante para evitar ressarcimentos ou indenizações por atrasos, gerando ônus para a empresa; Percentual de entrega do total de objetos (colunas DDOQ), que representa a capacidade de entrega dos objetos que chegam ao CDD,objetivando analisar e prevenir o acúmulo de objetos em uma determinada unidade, ocasionando perca de prazos; e Percentual de entrega em primeira tentativa (colunas EPTC), que analisa o percentual de objetos que foram entregues na primeira tentativa, ou seja, logo após o objeto chegar na unidade e ter seu primeiro evento de saída para entrega ao destinatário. Com este último indicador, objetiva-se evitar o repasse de trabalho para o dia posterior, ou seja, evitar o acúmulo de objetos que terão nova tentativa de entrega que, caso ocorra, serão somados aos objetos que chegam diariamente na unidade ocasionando uma sobrecarga de serviços aos colaboradores. Vale destacar que, para a modalidade "Telegrama", esse indicador não é verificado, uma vez que nessa modalidade existem várias saídas programadas no dia para esses objetos específicos.

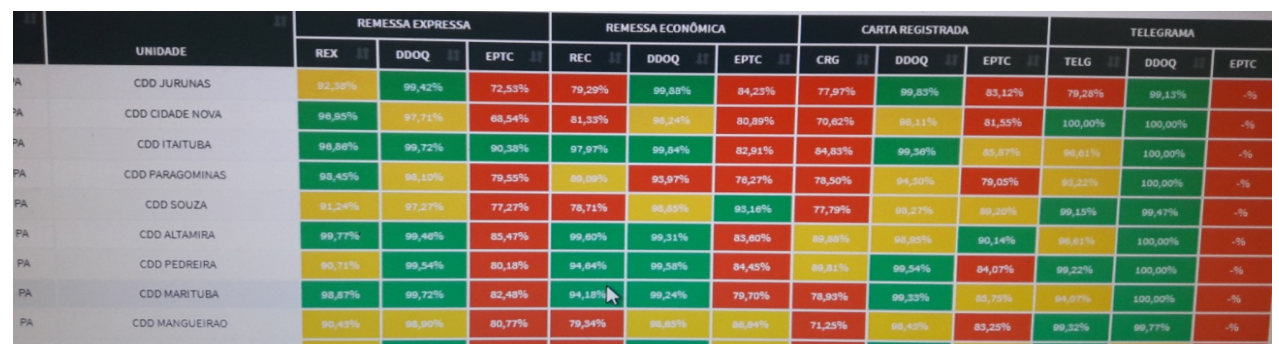

Figura 1. Relatório gerencial detalhado

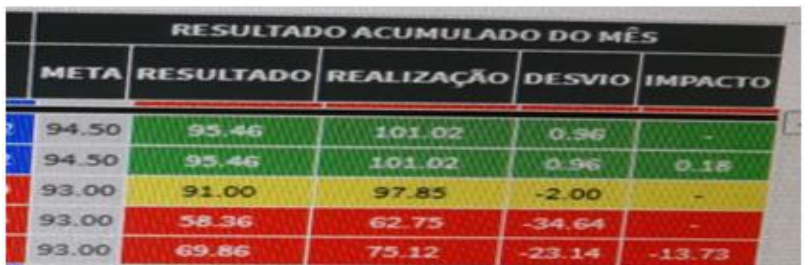

Figura 2. Relatório gerencial geral

Já o relatório gerencial geral apresenta vários indicadores (meta, resultado, realização, desvio e impacto), contudo para esse trabalho analisaremos apenas o resultado geral em comparação com a meta geral, que são calculados baseados nos indicadores descritos no relatório gerencial detalhado.

Assim, comparando as médias alcançadas no decorrer dos últimos 12 meses, com base nos relatórios gerenciais gerais, podemos observar a evolução da média alcançada pelo CDD Mangueirão, vide Figura 3, tendo como ápice o mês de junho do ano de 2019, seguida com uma acentuada queda de julho a setembro, culminando em uma grande recuperação no mês de outubro de 2019 (mês em que se aplicou o presente estudo de caso).

O mês de outubro de 2019 foi o de melhor desempenho, do segundo semestre, até a data do levantamento desses dados. Comparado com os três meses anteriores (julho, agosto e setembro), percebemos um resultado acima das médias alcançadas nesse período, com crescimentos de $3,15 \%, 0,75 \%$ e $2,10 \%$, respectivamente, o que nos leva a analisar o fator motivador dessa recuperação. $\mathrm{O}$ uso do software colaborativo Telegram, contribuiu para impulsionar os indicadores, alcançando média, no mês de outubro/2019, acima da meta estabelecida pela ECT e tendo um desempenho positivo se comparado com o mesmo período do ano anterior, vide Figura 4. 
Fazendo uma análise comparativa dos relatórios gerenciais detalhados nos meses de outubro/2018 e outubro/2019, vide Figura 4, percebemos a evolução dos indicadores nos diferentes anos para o mesmo mês, descartando a possibilidade desse resultado tratar-se de um fator histórico do período que viesse a se repetir anualmente.

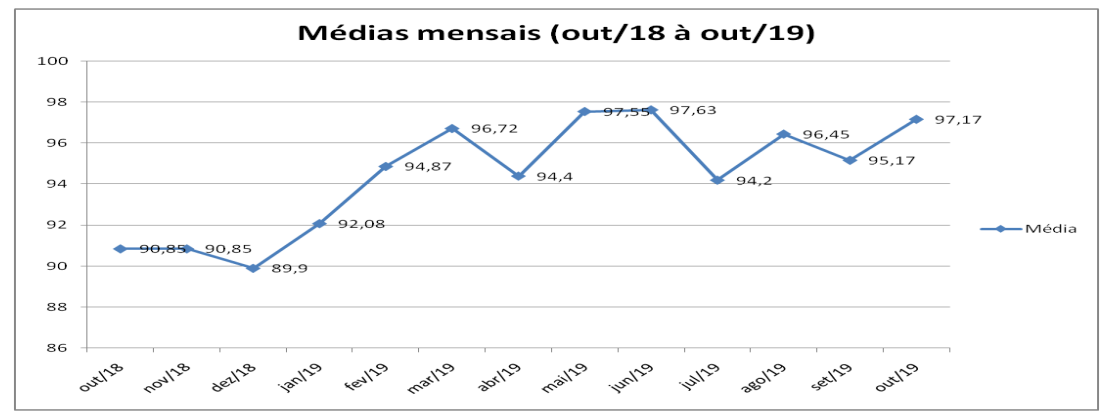

Figura 3. Médias alcançadas

Análise comparativa dos meses out/18 e out/19

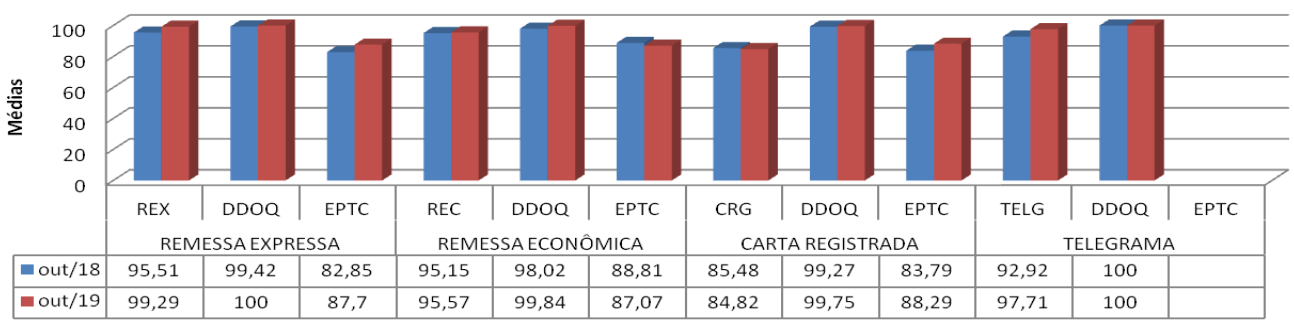

Figura 4. Análise comparativa do mês de outubro nos anos de 2018 e 2019

As médias alcançadas no mês de outubro/2019 superaram as médias alcançadas no mesmo período do ano anterior, exceto no quesito CRG (média de carta registrada entregue dentro do prazo). Um dos fatores apontados pelo gerente da unidade para a queda do indicador nesse quesito foi o fato de alguns objetos já terem chegado com o prazo vencido, ocasionado por alguma falha na logística geral da empresa, e que é contabilizado nos indicadores da unidade receptora desses objetos.

O teor das mensagens, áudio e ligações não foi analisado por não haver tempo hábil dentro dos processos da organização, ficando os smartphones condicionados a um conjunto de procedimentos para a finalização da operação diária e preparação para a próxima rotina diária, pelo setor responsável, quando o carteiro retorna da entrega. As informações extraídas da análise feita do uso do aplicativo Telegram pelos colaboradores participantes do estudo foram: ao longo do mês do estudo, 14 ligações entre os participantes, sendo 6 delas atendidas, usando a função de chamada do aplicativo Telegram; a duração média das ligações atendidas entre os participantes ficou em 51,6s, tempo mínimo de $1,23 \mathrm{~s}$ e tempo máximo de $60 \mathrm{~s}$; já a duração dos áudios enviados entre os participantes, ficou com um tempo médio de 14,7s, tempo mínimo de $2 \mathrm{~s}$ e tempo máximo de $29 \mathrm{~s}$, sendo trocados apenas 3 áudios entre os mesmos. Foram analisados os smartphones dos cinco participantes, uma única vez, ao final do período de trinta dias do estudo.

Algumas recomendações necessárias foram repassadas para os participantes, quanto ao uso do Telegram, de forma a evitar improdutividade e/ou possíveis colapsos na rede móvel do aparelho, ficando proibidos: o envio de vídeos, para não sobrecarregar a rede; as chamadas de videoconferências; eo diálogo paralelo via Telegram, durante o horário de trabalho, com pessoas que não estavam participando do estudo de caso afim de evitar impacto negativo na produtividade individual e coletiva dos participantes.

Vale lembrar que os participantes deste estudo de caso são especialistas em diferentes distritos e que o contato entre eles estava condicionado a tirar alguma dúvida ou dificuldade que viesse surgir na execução do trabalho, que necessitasse de uma informação ou 
conhecimento especializado. Além disso, foram trocadas várias mensagens por meio do aplicativo, entre os participantes do estudo de caso, o que reforça ainda mais o envolvimento dos mesmos no uso do software colaborativo e o empenho em alcançar as metas da unidade.

\subsection{Resultados Qualitativos}

Os resultados qualitativos foram coletados a partir de entrevistas na reunião de feedback com os participantes, onde foram analisadas a utilização do Telegram e suas funções. Foram apresentados como pontos fortes: a facilidade de comunicação, uma vez que cada participante não estaria "isolado" e "inacessível" no seu respectivo distrito; o fato da aplicação não ser usada anteriormente pelos participantes deste estudo, o que ajuda na concentração do trabalho, haja vista que outros contatos que não fossem do trabalho poderiam causar distrações aos participantes; e muito útil nos casos de urgências nos distritos, como por exemplo pneu furado, o que possibilitou acionar o socorro em tempo hábil sem mais prejuízos ou transtornos para o funcionário.

Como oportunidades foram citadas: a facilitação da entrega, onde foi possível consultar o especialista para identificar o endereço correspondente de determinada encomenda; e a possibilidade de criar grupo, facilitando a comunicação de grupos de interesses comuns. Já como ponto fraco foi citada a rede móvel utilizada, que tem um plano de dados bastante limitado, dificultando, em alguns momentos, a realização de chamadas.E, por fim, como ameaças, os participantes apontaram a possibilidade de influência externa (contatos pessoais), que viessem a existir ou mesmo aderir à utilização deste software colaborativo, podendo causar distrações com conversas paralelas, impactando na produtividade do funcionário.

\section{Conclusão}

Este trabalho foi fundamentado inicialmente por meio de pesquisas bibliográficas a fim de aprofundar o conhecimento sobre o conceito de conhecimento, gestão do conhecimento e utilização de software colaborativo como ferramenta de suporte em ambiente organizacional. Ao final da aplicação do estudo de caso foi possível responder a Questão de Pesquisa (QP), onde a utilização do software colaborativo Telegram contribui de forma positiva para a socialização do conhecimento, como pode ser constatada pelas análises qualitativa e quantitativa do estudo de caso, propiciando um ambiente favorável às interações sociais entre os participantes, mesmo geograficamente distantes, influenciando diretamente na eficácia dos processos organizacionais.

Algumas contribuições foram obtidas durante o desenvolvimento deste trabalho: a percepção da importância da gestão do conhecimento; e, a utilização do software colaborativo Telegram no processo de socialização do conhecimento em um CDD dos Correios, onde espera-se que este estudo de caso auxilie pesquisadores no uso de softwares colaborativos em empresas públicas e privadas, principalmente no processo de gestão do conhecimento.

Como limitações têm-se as principiais: a rede de dados móvel utilizada na empresa, que tem capacidade limitada para o atendimento às demandas previamente estabelecida pela organização; a proibição do uso de algumas funcionalidades do Telegram, tais como vídeo chamadas; o tempo limitado de acesso aos smartphones para realização de análises mais detalhadas, haja vista o procedimento necessário de finalização e preparação dos aparelhos para a rotina diária de trabalho; o fato que não foram usados comparativos entre dois CDD, onde em apenas um seria adotado o uso do Telegram, com a finalidade de medir e eficiência dessa ferramenta no processo de socialização do conhecimento; e a quantidade de participantes do estudo de caso, necessitando uma avaliação com um número mais expressivo de usuários afim de verificar a eficiência do software colaborativo Telegram frente a essa demanda. 
Por fim, como trabalhos futuros apontamos: a aplicação deste estudo de caso com um número expressivo de participantes; uma análise comparativa entre os diferentes CDDs; e a possibilidade de usar a API do Telegram para criar ferramentas visando facilitar a gestão dos smartphones, no sentido de coleta dos dados no final do dia (número de ligações; mensagens trocadas; quantidade de áudios trocados; etc.).

\section{Agradecimentos}

Este trabalho foi elaborado durante a disciplina de Softwares Colaborativos do PPGCC/UFPA, ministrada pelo Prof. Dr. Cleidson de Souza, o qual os autores são gratos pelos ensinamentos.

\section{Referências}

Aires, R. W.do A., Kempner-Moreira, F., Freire, P. de S. (2017) "Indústria 4.0: Desafios e tendências para a Gestão do Conhecimento", I SUCEG. Santa Catarina.

Almenara, J.C., Osuna, J. B.,Cejudo, M. C. L., Cabrera, C. Y. (2016) "Redes sociales y Tecnologías de la Información y la Comunicación en Educación: aprendizaje colaborativo, diferencias de género, edad y preferencias”, Revista de Educación a Distancia. N. 51. A. 1.

Braquehais, A. de P., Wilbert, J. K. W., Moresi, E. A. D., Dandolini, G. A. (2017) "O Papel da Cultura Organizacional na Gestão do Conhecimento: Revisão de Literatura de 2009 A 2015", Perspectivas em Gestão \& Conhecimento, v. 7, Número Especial, p. 80-93.

Correa, F., Ziviani, F., Chinelato, F. B. (2017) "Tipos e usos de ferramentas de apoio a gestão de conhecimento em uma empresa de Tecnologia da Informação", Encontros Bibli: revista eletrônica de biblioteconomia e ciência da informação, v. 22, n.48, p. 27-40.

Correios (2019) "A Empresa", Acesso em 03 de dezembro de 2019. Disponível em: http://www.correios.com.br/sobre-os-correios/a-empresa.

Ministério da Ciência, Tecnologia, Inovações e Comunicações (MCTIC). (2016) "Aprimoramento da prestação dos serviços postais básicos pelo ECT", Gabinete do Ministro. Portaria n ${ }^{\circ}$ 6.194, de 27 de dezembro de 2016.

Ministério da Ciência, Tecnologia, Inovações e Comunicações (MCTIC). (2018) "Diretrizes e metas para a implantação da Distribuição Domiciliária Alternada na ECT", Gabinete do Ministro. Portaria n ${ }^{0} 1.203$, de 7 de março de 2018.

Pimentel, M. A. da S., Santos, V. C., Silva, F. A. O. da, Gonçalves, A. C. (2012). "A Ocupação das Várzeas na Cidade de Belém: Causas e Consequências Socioambientais", Revista GEONORTE, Edição Especial, V.2, N.4, p.34 - 45.

Santos, M. de A., Grechi, J. G., Bermejo, P. H. de S. (2010). “Avaliação do Impacto do SCRUM no desenvolvimento de software utilizando a análise SWOT”, In: XXX ENEGEP. São Paulo.

Schafermeyer, R. G. e Hoffman, R. R. (2016). "Using knowledge libraries to transfer expert knowledge", IEEE Intell.Syst. 31(2), 89-93.

Sehn, M. F., Reis, R. A. dos, Silva, H.de F. N. da. (2013). "Panorama Nacional das Pesquisas Sobre a Gestão do Conhecimento: Um Estudo a Partir da Plataforma Scielo", Revista Latino-Americana de Inovação e Engenharia de Produção Vol. 1, n. 1.

Silva, L. C., Damian, I. P. M., Segundo, J. E. S. (2016). "Melhores práticas para aplicação de projetos de gestão do conhecimento: instituindo ambientes colaborativos". Biblos: Revista do Instituto de Ciências Humanas e da Informação, v. 30, n.1.

Souza, L. M. e Heis, A. (2017) "A utilização de Ambientes Virtuais de Ensino e Aprendizagem como ambientes colaborativos em uma empresa", Trabalho de Curso Superior de Tecnologia em Gestão da Tecnologia da Informação, IFSC.

Tabares, M. S., Giraldo, L., Joyanes, L. (2016) "Improving the Business Processes Management from the Knowledge Management", 11th KMO.

Telegram.org. (2019). "Perguntas frequentes", Acesso em 02 de dezembro de 2019. Disponível em: https://telegram.org/faq/br. 